\title{
Necrose Etendue De L'estomac Sur Dilatation Gastrique Chez Un Patient Suivi Pour Tumeur Cerebelleuse
}

\author{
Davidà S. Rakotomena \\ Aurélia Rakotondrainibe \\ Gabrielle E.P. Andrianah \\ Tovohery A. Rajaonera \\ Hery N. Rakoto-Ratsimba
}

Faculty of Medicine of Antananarivo,

University of Antananarivo, Madagascar

Doi: 10.19044/esj.2018.v14n27p21 URL:http://dx.doi.org/10.19044/esj.2018.v14n27p21

\begin{abstract}
Acute necrosis with a gastric dilatation is very rare. It usually occurs on psychiatric and neurological field. The preoperative diagnosis is difficult, and it is often revealed by radiological exams. With or without surgery, mortality remains high. We report a case of gastric necrosis in a patient with a severe neurological history. The clinical signs were vomiting, without intestinal transit disorders, and poor general condition. The radiological exams revealed gastric gas gangrene in the stomach wall. The surgical management determined the limit of gastric necrosis and gastrectomy was performed.
\end{abstract}

Keywords: Gastric dilatation, Necrosis, Radiology, Stomach, Surgical treatment.

\section{Resume}

La nécrose aiguë de l'estomac est une pathologie très rare, compliquant parfois une dilatation gastrique laquelle survient habituellement sur un terrain neuro-psychiatrique. Le diagnostic est difficile en préopératoire et il est souvent radiologique. La mortalité est élevée avec ou sans chirurgie. Nous rapportons un cas de nécrose gastrique chez un patient avec de lourds antécédents neurologiques. Les signes d'appel étaient des vomissements sans trouble du transit intestinal et une altération de l'état général. L'imagerie était en faveur d'une gangrène gazeuse gastrique. L'intervention chirurgicale avait permis de déterminer la limite de la nécrose gastrique et avait abouti à une 
gastrectomie partielle.

Mots clés: Dilatation gastrique, Estomac, Nécrose, Radiologie, Traitement chirurgical.

\section{Introduction}

La riche vascularisation de l'estomac, composée de cinq sources artérielles collatérales majeures, de six mineures et de nombreux systèmes anastomotiques qui les relient, fait que la nécrose aiguë de l'estomac est une pathologie très rare (Sahoo et al., 2013 ; Tiwari et al., 2010). Il s'agit d'une complication inhabituelle de la dilatation aiguë de l'estomac (Gomez et al., 2004).

Nous rapportons un cas d'ischémie gastrique sur dilatation aiguë de l'estomac chez un patient avec des séquelles neurologiques d'une tumeur cérébelleuse dans l'enfance.

\section{Observation}

Il s'agissait d'un jeune homme de 37 ans, grabataire, admis pour vomissements sans trouble du transit intestinal dans un contexte apyrétique. Dans ses antécédents étaient retenus une dérivation ventriculo-péritonéale pour tumeur cérébelleuse dans l'enfance avec des séquelles neurologiques à type d'encéphalopathie et d'épilepsie résistante au traitement et de cyphoscoliose. A l'admission à l'hôpital, l'examen avait révélé un état hémodynamique stable, néanmoins associé à une altération de l'état général et une défense généralisée de l'abdomen. Le toucher rectal avait objectivé un cul de sac de Douglas libre mais douloureux ; le doigtier avait ramené du sang noirâtre. Lors de l'exploration radiologique, le scanner abdominal avait révélé un pneumopéritoine associé à une pneumatose pariétale diffuse d'un estomac distendu évoquant une gangrène gazeuse gastrique, une dilatation des premières anses grêles sans obstacle apparent en aval, une aéroportie et la présence du cathéter de dérivation ventriculo-péritonéale en intra-abdominal (Figures 1 et 2). 


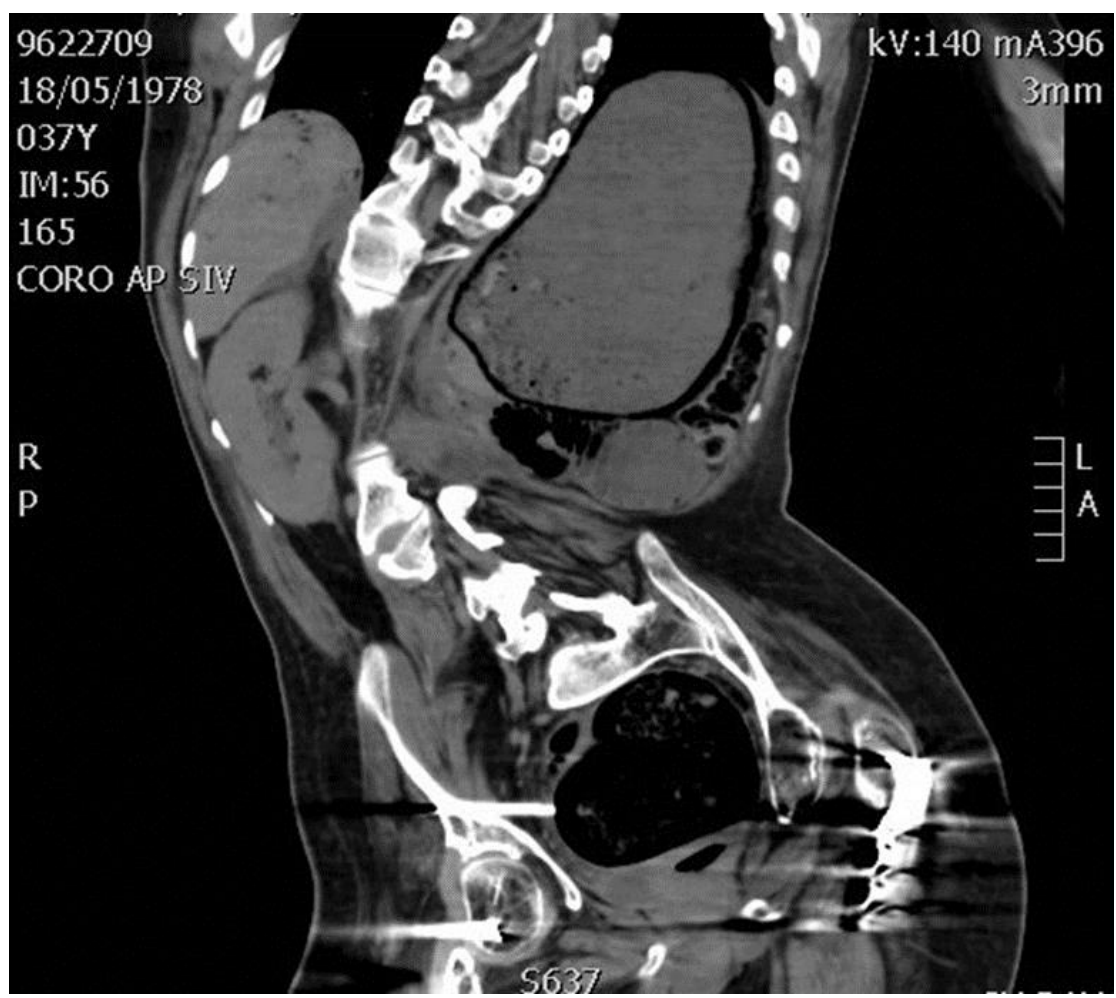

Figure 1 : Pneumatose gastrique diffuse sur un estomac distendu, associée à une aéroportie sur une coupe coronale du scanner abdominal. Une cyphose est également constatée.

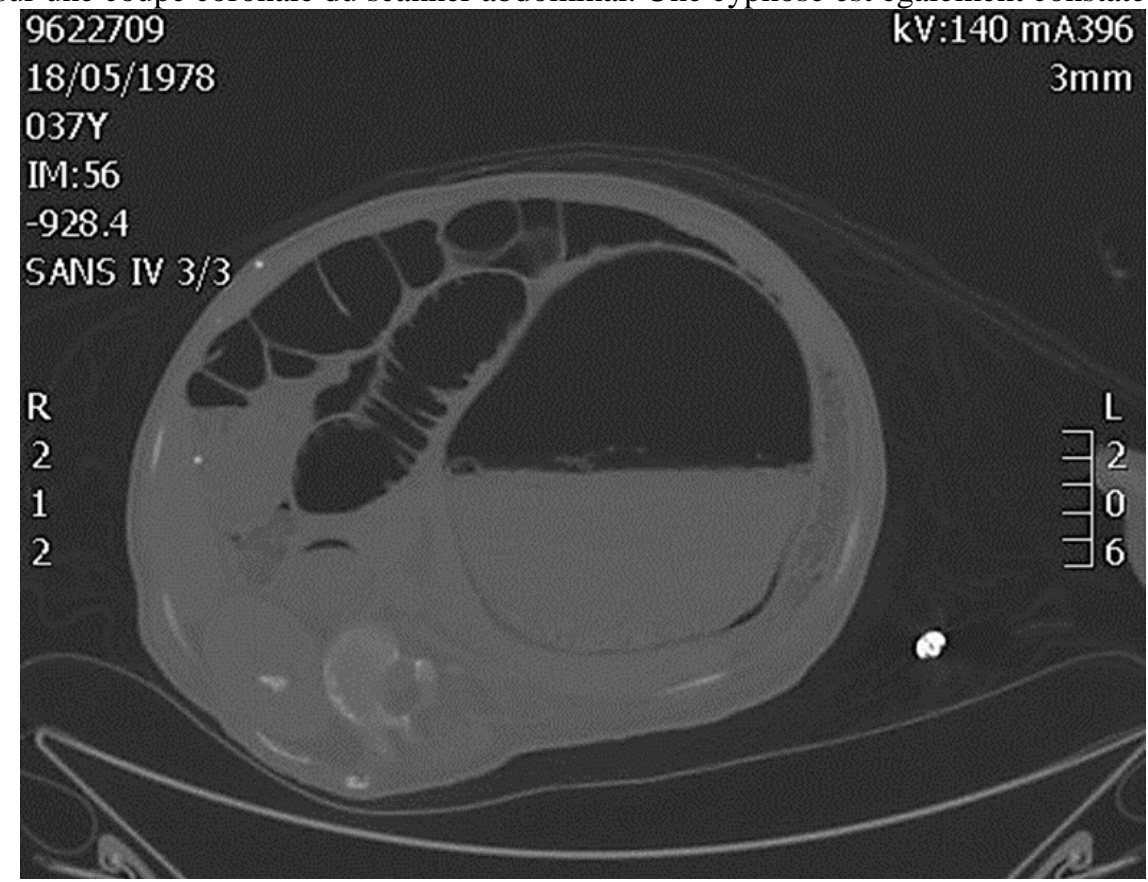

Figure 2 : Distension gastrique et des premières anses grêles associée à une pneumatose de la face postérieure de l'estomac, sur une coupe axiale du scanner abdominal. 
Après une réanimation pré-opératoire, une laparotomie, sous anesthésie générale, était effectuée en urgence et avait révélé un estomac distendu avec nécrose diffuse de la paroi postérieure (Figures 3 et 4), sans signe d'infarctus de la rate lors de l'exploration des autres organes.

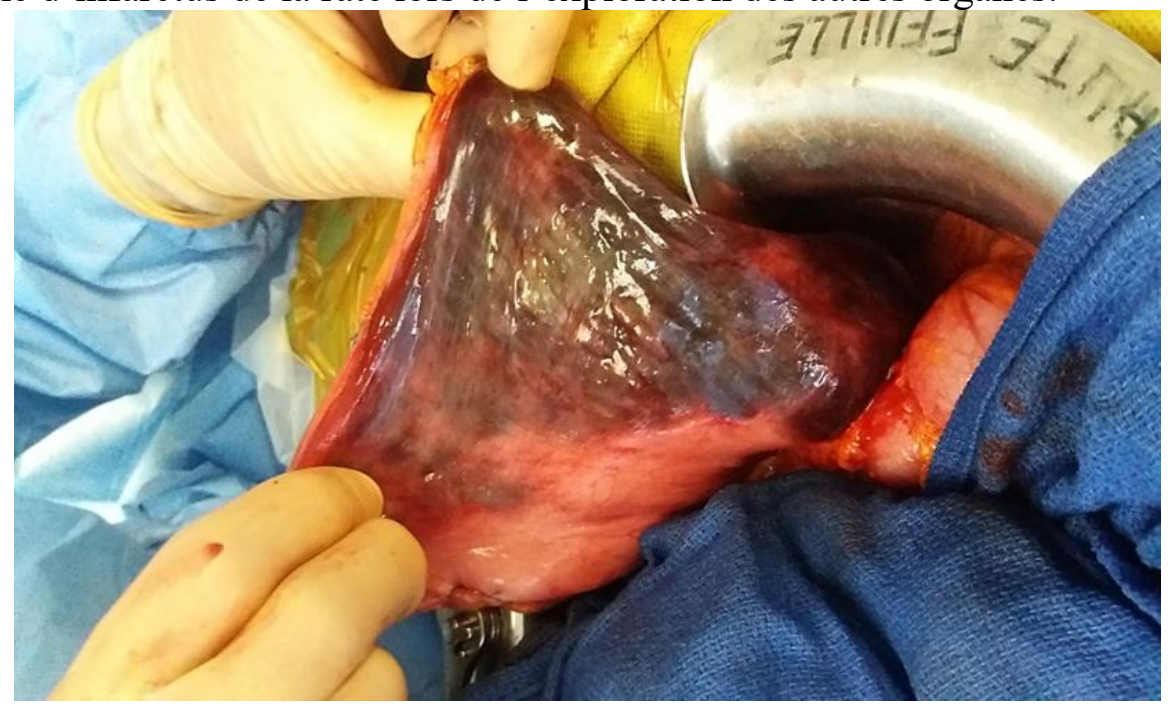

Figure 3 : Vue peropératoire : Nécrose étendue de la face postérieure de l'estomac.

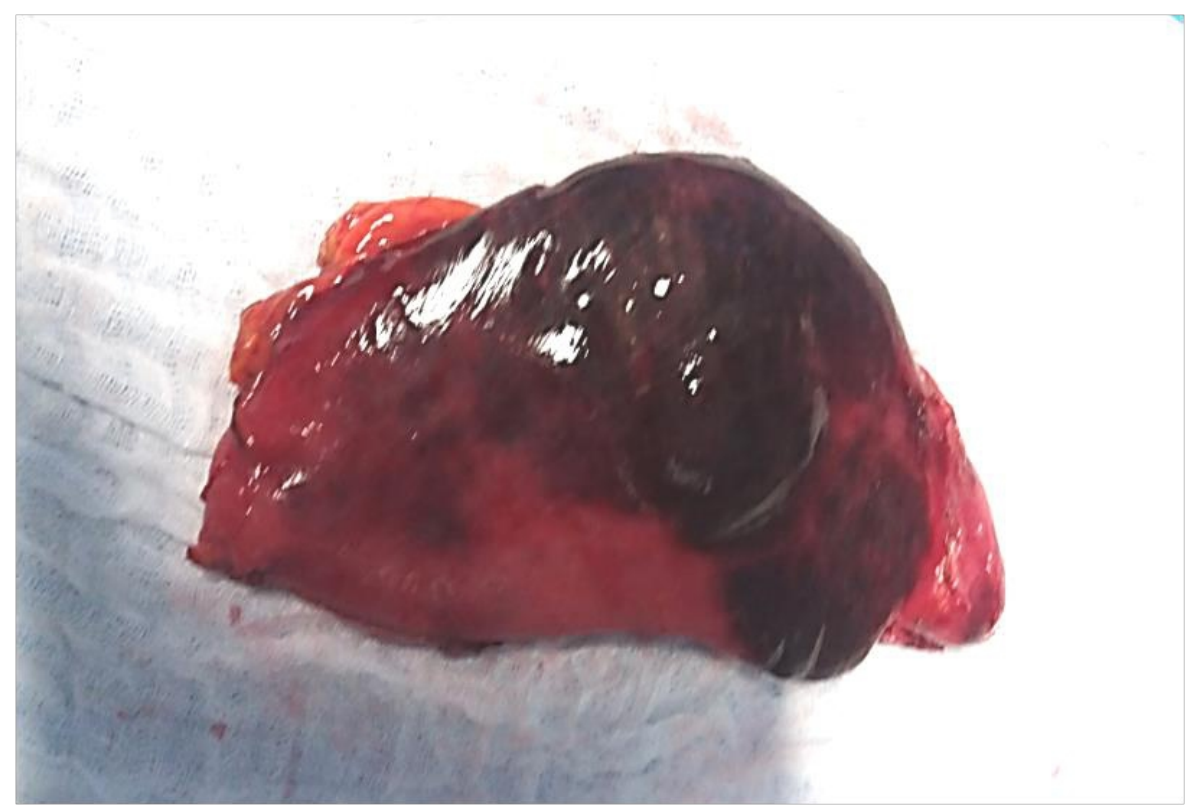

Figure 4 : Pièce opératoire de la gastrectomie partielle atypique.

Une gastrectomie partielle atypique était décidée suivie d'une anastomose gastro-jéjunale en Y de Roux. L'examen anatomopathologique de la pièce opératoire avait confirmé une ischémie partielle de la paroi gastrique 
sans découverte d'embole à la limite de l'examen. Les suites opératoires étaient simples, après optimisation hydro-éléctrolytique et nutritionnelle postopératoire. Le patient était autorisé à sortir de l'hôpital au dixième jour postopératoire.

\section{Discussion}

La dilatation aiguë de l'estomac intéresse toutes les races à tout âge, mais touche surtout les patients âgés de 60 à 70 ans et le genre féminin dans 35 à 37\% des cas, (Park et al., 2017 ; Sharma et al., 2017 ; Touré et al., 2005). Une nécrose gastrique, partielle ou totale, peut la compliquer par insuffisance veineuse pariétale, et habituellement, sur un terrain neuropsychiatrique particulier (Sahoo et al., 2013 ; Touré et al., 2005) (Tirawi et al. 2010). Mais elle peut être aussi secondaire à une maladie athéromateuse du tronc cœliaque où un infarctus splénique est souvent associé (Park et al., 2010 ; Sharma et al., 2017), à un volvulus gastrique, à une hernie intrathoracique avec strangulation gastrique, à une gastrite caustique ou à un traumatisme abdominal (Tirawi et al., 2010). Elle intéresse la face antérieure de l'estomac dans $40 \%$ des cas, la grande courbure dans $23 \%$ des cas et la face postérieure ou la petite courbure dans $15 \%$ des cas (Ourfali et al., 2010). Notre cas concernait une nécrose de la paroi postérieure gastrique par dilatation aiguë de l'estomac chez un patient suivi pour une tumeur cérébelleuse opéré.

Rarement évoquée en préopératoire, la distension gastrique évoque souvent un syndrome occlusif haut retardant son diagnostic au stade de complication (Sahoo et al., 2013 ; Sharma et al., 2017 ; Touré et al., 2005). En effet, la dilatation gastrique aiguë est secondaire à une paralysie de 1'estomac par inhibition réflexe des mécanismes moteurs de cet organe (Leigh, 1960). Elle peut avoir plusieurs étiologies - dont certaines peuvent entraîner une atonie associée à une amyotrophie musculaire de l'estomac - ; étiologies comme une anorexia nervosa (caractérisée par une diminution de la vidange gastrique liée à une paralysie gastrique neurogénique secondaire à une malnutrition), une boulimie psychogénique, une longue période de jeûne, une ingestion brutale et massive d'aliments, un volvulus gastrique (Abdu et al., 1987 ; Luncă S et al., 2005). De plus des facteurs prédisposants (tels les dysfonctions ou déformations de la colonne vertébrale, la convalescence, l'altération de l'état général, le faible indice de masse corporelle, les procédures chirurgicales au niveau de l'abdomen, ...) favorisent cette dilatation (Leigh T, 1960 ; Luncă S et al., 2005). Une nécrose des parois gastriques se produit alors lorsque la pression intragastrique dépasse la pression gastrique veineuse (Abdu et al., 1987). Les antécédents de pathologies neurologiques associés à la présence de cyphoscoliose du patient de notre cas reporté auraient pu alors être un facteur favorisant de troubles alimentaires à type d'anorexie ou de malnutrition (devant 1'altération de l'état 
général et l'état grabataire de celui-ci) ayant pour conséquence la dilatation gastrique. Cette dilatation ayant conduit à la nécrose gastrique, cependant sans signe évocateur et non spécifique de cette dernière. En effet, des auteurs rapportaient des tableaux cliniques non spécifiques : douleurs abdominales $(65 \%)$, saignements gastro-intestinaux $(47 \%)$, altération de l'état mental (23\%), signes de choc, vomissements, syndrome de compression de la veine cave inférieure, avec une distension abdominale constante par la dilatation aiguë de l'estomac (Sharma et al., 2017 ; Perpresse et al., 2013 ; Touré et al., 2005). La nécrose gastrique est suspectée devant la distension abdominale associée à un tympanisme diffus et une crépitation gazeuse au niveau de l'hypogastre et du flanc gauche (Sahoo et al., 2013). Chez notre patient, la découverte de la dilatation était concomitante à celle de la nécrose gastrique, lors de l'exploration radiologique, sur un tableau clinique trompeur constitué de vomissements avec méléna, dans un contexte apyrétique associée à une péritonite aiguë généralisée dans un tableau d'altération de l'état général. Ainsi, l'imagerie est très contributive dans le diagnostic. En effet, l'abdomen sans préparation peut visualiser une volumineuse poche à air gastrique pouvant s'étendre sur les hypochondres associée à une pneumatose sous forme d'une clarté linéaire soulignant la lumière de 1'estomac (Sahoo et al., 2013 ; Touré et al., 2005; Gomez et al. 2004). Comme dans notre cas, la tomodensitométrie abdominale a confirmé le diagnostic devant un estomac distendu, sans prise de contraste de la paroi gastrique, mais accompagné d'images de pneumatoses et éventuellement d'une aéroportie (Touré et al., 2005 ; Gomez et al., 2004 ; Sharma et al., 2017). En outre, l'endoscopie pourrait visualiser directement une muqueuse gastrique nécrotique décolorée avec éventuellement de grandes ulcérations confluentes (Park et al., 2010).

La prise en charge est initiée par des mesures de réanimation basées essentiellement sur la mise en place d'une sonde gastrique en aspiration douce pour assurer la décompression de l'estomac, la correction de l'état hémodynamique et des troubles hydro-électrolytiques (Sahoo et al., 2013 ; Touré et al., 2005 ; Perpresse et al., 2013 ; Moslim et al., 2017). Cette première prise en charge médicale sera suivie d'emblée de l'intervention chirurgicale dont 1'exploration chirurgicale confirme la dilatation importante de l'estomac et précise la localisation exacte et l'étendue de la nécrose gastrique, ainsi que la taille d'une éventuelle rupture gastrique (Sahoo et al., 2013 ; Touré et al., 2005). Au bilan des lésions dépend le choix du geste chirurgical allant d'une simple suture à une gastrectomie atypique, voire une gastrectomie totale (Sahoo et al., 2013 ; Touré et al., 2005 ; Moslim et al., 2017).

Bien que rarement évoqué en préopératoire, le diagnostic de la dilatation aiguë de l'estomac doit être précoce car sa morbi-mortalité élevée dans les formes compliquées, particulièrement, en cas d'ischémie gastrique en raison de sa fréquente méconnaissance et de son caractère brutal (Sahoo et al., 
2013 ; Tirawi et al., 2010 ; Sharma et al., 2017). Sans chirurgie, la mortalité de l'ischémie gastrique est évaluée à $80 \%$ à $100 \%$, et elle est de 50 à $65 \%$ après chirurgie (Gomez et al., 2004 ; Touré et al., 2005).

\section{Conclusion}

La nécrose gastrique devrait faire partie des diagnostics à suspecter devant un tableau abdominal aigu, bien que les signes ne soient pas toujours évidents en pré-opératoire. Dans notre cas, le patient avait des antécédents notables neurologiques et une cyphoscoliose qui auraient pu contribuer à la dilatation gastrique ayant évolué vers la nécrose de l'estomac. La prise en charge repose essentiellement sur la chirurgie en urgence, associée à des mesures de réanimation adéquates. Cette prise en charge doit être rapide car la mortalité reste élevée.

\section{Conflit d'interet}

Les auteurs ne déclarent aucun conflit d'intérêt.

\section{References:}

1. Abdu, R.A., Garritano, D., \& Culver, O. (1987). Acute gastric necrosis in anorexia nervosa and bulimia. Two case reports. Archives of Surgery (Chicago, Ill.: 1960), 122(7), 830-832.

2. Gomez, M.A., Blum, L., Scotto, B., Besson, M., Roger., R., \& Alison, A. (2004). Nécrose gastrique sur distension aiguë sans contexte psychiatrique. Journal de Radiologie, 85(5 Pt 1), 643-645.

3. Leigh TF. (1960). Acute gastric dilatation. Journal of the American Medical Association, 26, 172, 1376-1381.

4. Luncă, S., Rikkers, A., Stănescu, A. (2005). Acute massive gastric dilatation: severe ischemia and gastric necrosis without perforation. Romanian Journal of Gastroenterology, 14(3), 279-283.

5. Moslim, M.A., Mittal, J., Falk, G.A., Ustin, J.S., \& Morris-Stiff, G. (2017). Acute massive gastric dilatation causing ischaemic necrosis and perforation of the stomach. BMJ Case Reports, 15, 2017, pii: bcr2016-218513.

6. Ourfali, N., Ali, A.M., Tokhais, T., Hadad, M., \& Hassab, M.H. (2010). Idiopathic gastric rupture in a child : critical situation. Annals of Pediatric Surgery, 6, 1, 44-46.

7. Park, H.H., Lee, H.S., Kim, J.S., Kang, S.H., Moon, H.S., Sung, J.K., Jeong, H.Y., \& Sul, J.Y. (2017). Ischemic necrosis of the gastric remnant without splenic infarction following subtotal gastrectomy. Clinical Endoscopy, 24. 
8. Pepresse, X., Bodson, L., \& Au, S.M. (2013). Gastric dilatation and circulatory collapses due to eating disorder. American Journal of Emergency Medicine, 31(3), 633.e3-4.

9. Sahoo, M.R., Kumar, A.T., Jaiswal, S., \& Bhujabal, S.N. (2013). Acute dilatation, ischemia, and necrosis of stomach without perforation. Case Report in Surgery, 2013, 2013, 984594.

10. Sharma, A., Mukewar, S., Chari, S.T., \& Wong Kee Song, L.M. (2017). Clinical features and outcomes of gastric ischemia. Digestive Diseases and Sciences, 62(12), 3550-3556.

11. Tiwari, N., Sharma, A.K., Galagali, A., Kumar, M., \& Chand, K.. (2010). Acute idiopathic gastric gangrene with perforation. Tropical Gastroenterology, 31(4), 339-341.

12. Touré, A.O., Thiam, O., Cisée, M., Nduwimana, D., Gueye, M.L., Seck, M., Ka, O., Dieng, M., \& Touré, C.T. (2005). Dilatation aiguë de l'estomac: à propos de 02 cas et revue de la littérature. Pan African Medical Journal, 4, 22, 210-215. 